\title{
A!
}

This is an electronic reprint of the original article.

This reprint may differ from the original in pagination and typographic detail.

Hu, Xuerong; Du, Luojun; Wang, Yadong; Lahtinen, Jouko; Yao, Lide; Ren, Zhaoyu; Sun, Zhipei

Raman fingerprints and exciton-phonon coupling in 2D ternary layered semiconductor InSeBr

Published in:

Applied Physics Letters

DOI:

$10.1063 / 1.5143119$

Published: 20/04/2020

Document Version

Publisher's PDF, also known as Version of record

Please cite the original version:

Hu, X., Du, L., Wang, Y., Lahtinen, J., Yao, L., Ren, Z., \& Sun, Z. (2020). Raman fingerprints and excitonphonon coupling in 2D ternary layered semiconductor InSeBr. Applied Physics Letters, 116(16), [163105]. https://doi.org/10.1063/1.5143119

This material is protected by copyright and other intellectual property rights, and duplication or sale of all or part of any of the repository collections is not permitted, except that material may be duplicated by you for your research use or educational purposes in electronic or print form. You must obtain permission for any other use. Electronic or print copies may not be offered, whether for sale or otherwise to anyone who is not an authorised user. 


\section{Raman fingerprints and exciton- phonon coupling in 2D ternary layered semiconductor InSeBr}

Cite as: Appl. Phys. Lett. 116, 163105 (2020); https://doi.org/10.1063/1.5143119

Submitted: 21 December 2019 . Accepted: 07 April 2020. Published Online: 24 April 2020

Xuerong Hu (D), Luojun Du (D), Yadong Wang (D), Jouko Lahtinen (D), Lide Yao (D), Zhaoyu Ren, and Zhipei Sun (iD)
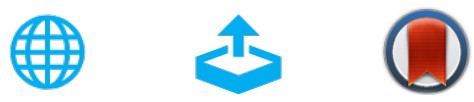

View Online

Export Citation

\section{ARTICLES YOU MAY BE INTERESTED IN}

Terahertz emission from in-plane and out-of-plane dipoles in layered SnS 2 crystal

Applied Physics Letters 116, 161901 (2020); https://doi.org/10.1063/5.0004701

High performance $\mathrm{CsPbBr}_{3}$ quantum dots photodetectors by using zinc oxide nanorods arrays as an electron-transport layer

Applied Physics Letters 116, 162103 (2020); https://doi.org/10.1063/5.0005464

Low voltage thin film transistors based on solution-processed $\operatorname{In}_{2} \mathrm{O}_{3}: \mathrm{W}$. A remarkably stable semiconductor under negative and positive bias stress

Applied Physics Letters 116, 163505 (2020); https://doi.org/10.1063/1.5142699

\section{Lock-in Amplifiers up to 600 $\mathrm{MHz}$}
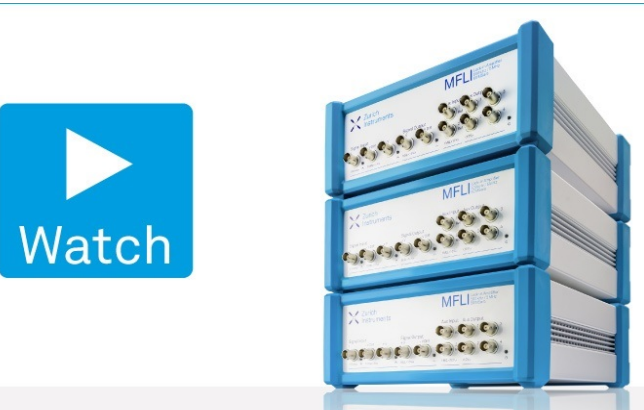


\title{
Raman fingerprints and exciton-phonon coupling in 2D ternary layered semiconductor InSeBr
}

Cite as: Appl. Phys. Lett. 116, 163105 (2020); doi: 10.1063/1.5143119

Submitted: 21 December 2019 . Accepted: 7 April 2020 .

Published Online: 24 April 2020

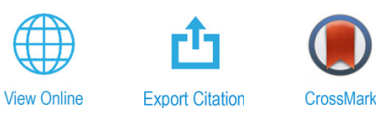

Xuerong Hu, ${ }^{1,2}$ (D) Luojun Du, ${ }^{2, a)}$ (iD Yadong Wang, ${ }^{2}$ (D) Jouko Lahtinen, ${ }^{3}$ (DD Lide Yao, ${ }^{3}$ (iD) Zhaoyu Ren, ${ }^{1, b)}$ and Zhipei Sun ${ }^{2,4}$ (iD

\author{
AFFILIATIONS \\ ${ }^{1}$ Institute of Photonics and Photon Technology, Northwest University, Xi'an 710069, China \\ ${ }^{2}$ Department of Electronics and Nanoengineering, Aalto University, Espoo 02150, Finland \\ ${ }^{3}$ Department of Applied Physics, Aalto University, Espoo 02150, Finland \\ ${ }^{4}$ QTF Centre of Excellence, Department of Applied Physics, Aalto University, Espoo 00076, Finland \\ a)|uojun.du@aalto.fi \\ b) rzy@nwu.edu.cn
}

\begin{abstract}
Compared to other two-dimensional (2D) crystals with single or binary elements, 2D ternary layered materials have unique physical properties for potential applications due to the stoichiometric variation and synergistic effect. Here, we report the first investigation of lattice dynamics and interactions between the exciton and lattice degrees of freedom in a 2D ternary semiconductor: indium-selenide-bromide (InSeBr). Via linear polarization resolved Raman scattering measurements, we uncover three Raman modes in few-layer InSeBr, including two $\mathrm{A}_{1 \mathrm{~g}}$ and one $\mathrm{E}_{\mathrm{g}}$ modes. Moreover, through the combination of temperature-dependent Raman scattering experiments and theoretical calculations, we elucidate that few-layer InSeBr would harbor strong coupling between excitons and phonons. Our results may provide a firm basis for the development and engineering of potential optoelectronic devices based on 2D ternary semiconductors.
\end{abstract}

Published under license by AIP Publishing. https://doi.org/10.1063/1.5143119

Since the discovery of graphene, ${ }^{1}$ two-dimensional (2D) van der Waals (vdW) materials have sparked significant attention and initiated the promising era of basic scientific research and modern technological advances because of their unique electrical, optical, and magnetic properties. ${ }^{2-9}$ For example, $2 \mathrm{D}$ honeycomb lattices, such as gapped graphene and transition metal dichalcogenides (TMDCs), reinvigorate the field of valleytronics and provide an unprecedented platform to address and control the valley degrees of freedom for information storage and encoding. ${ }^{3,8,10-14}$ Until now, the research of $2 \mathrm{D}$ materials is mainly focused on single (for example, graphene, ${ }^{1,15}$ black phosphorus, ${ }^{16,17}$ and silicene ${ }^{18,19}$ ) and binary elemental crystals (such as TMDCs, ${ }^{3,20,21}$ $\mathrm{GaSe}^{22} \mathrm{GeSe}^{23}$ and $\mathrm{InSe} \mathrm{St}^{24,25}$ ).

Strikingly, the recently emerging $2 \mathrm{D}$ ternary vdW materials have attracted increasing attention due to their enchanting physical properties for potential applications since the inclusion of a third element offers one more degree of freedom to control the stoichiometry and tune the physical properties. ${ }^{26-31}$ For instance, the ferromagnetic transition temperature of itinerant vdW ferromagnet $\mathrm{Fe}_{3} \mathrm{GeTe}_{2}$ can be tuned from $150 \mathrm{~K}$ to $220 \mathrm{~K}$ by changing the Fe occupancy. ${ }^{32}$ Few-layer $2 \mathrm{D}$ ternary $\mathrm{Ga}_{2} \mathrm{In}_{4} \mathrm{~S}_{9}$ exhibits appealing ultraviolet light detection performance with an outstanding responsivity of $111.9 \mathrm{AW}^{-1}$, an excellent external quantum efficiency of $3.85 \times 10^{4} \%$, and a specific detectivity of $2.25 \times 10^{4}$ Jones. ${ }^{30}$ Ultrathin $\mathrm{Bi}_{2} \mathrm{O}_{2}$ Se nanosheets provide high electron mobility $\left(\approx 30000 \mathrm{~cm}^{2} \mathrm{~V}^{-1} \mathrm{~s}^{-1}\right.$ at low temperature), a moderate bandgap $(\approx 0.8 \mathrm{eV})$, a near-ideal subthreshold swing value

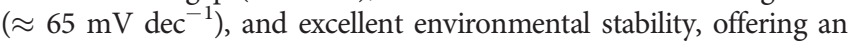
unprecedented platform toward high-performance optoelectronic devices at the nanoscale. ${ }^{27,33-35}$ In addition, 2D ternary materials can harbor the synergistic effect between various components, giving rise to fascinating phenomena and functionalities. For example, by adding $\mathrm{Fe}^{3+}$ ions into $\mathrm{CuSe}$, forming $\mathrm{CuFeSe}$ nanosheets, the intrinsic properties and performance could be highly modified, which shows great promise of ternary metal chalcogenide nanosheets for chemotherapy of cancer and exhibits excellent anticancer efficacy. ${ }^{36}$

The studies of $2 \mathrm{D}$ ternary vdW materials are still at the infant stage, and so it is important to search for potential ternary vdW materials and investigate the corresponding basic physical properties, such as phonon-phonon and electron-phonon coupling, which play a vital role in a wide range of applications in condensed matter physics. ${ }^{37-41}$ InSeBr, a ternary layered semiconductor with a deep-red color and 
energy bandgap in the visible region, ${ }^{42,43}$ would open a promising paradigm for a variety of potential applications in the fields of electronics and optoelectronics. However, the properties of InSeBr are largely unexplored. Here, we systematically and comprehensively investigate the phonon behaviors and exciton-phonon coupling in few-layer InSeBr. We uncover the Raman fingerprints (two $A_{1 g}$ and one $E_{g}$ ) of few-layer InSeBr by polarized Raman scattering experiments. Moreover, the temperature-dependent Raman measurements show that the evolution of phonon lifetimes with temperature strongly diverges from the anharmonic model. In conjunction with photoluminescence (PL) spectra and theoretical calculations, we elucidate that the increase in phonon linewidths with decreasing temperature can be understood as the strong coupling between phonons and excitons in few-layer InSeBr.

Ternary InSeBr is a layered material with weak vdW interaction and reveals p-type semiconducting behavior. ${ }^{43,44}$ Each layer is threeatom thick, where the In atoms of the middle sheet are sandwiched between a single layer of $\mathrm{Se}$ and $\mathrm{Br}$ atoms in a trigonal structure $\left(\mathrm{a}, \mathrm{b}=0.40 \mathrm{~nm}, \mathrm{c}=1.88 \mathrm{~nm}\right.$, and $\alpha, \beta=90^{\circ}, \gamma=120^{\circ}$ ), as shown in Fig. 1(a) (top view) and 1(b) (side view) ${ }^{42,43}$ In order to confirm the chemical composition and quality of the bulk sample (2D Semiconductors), we performed the $\mathrm{x}$-ray photoelectron spectroscopy (XPS) measurements (see supplementary material S1). The binding energies of In $3 \mathrm{~d}_{5 / 2}$ and In $3 \mathrm{~d}_{3 / 2}$ correspond to $452.4 \mathrm{eV}$ and $444.9 \mathrm{eV}$, respectively [Fig. 1(c)]. The Se $3 \mathrm{~d}$ and $\mathrm{Br} 3 \mathrm{~d}$ peaks are deconvoluted to show the $3 \mathrm{~d}_{5 / 2}$ and $3 \mathrm{~d}_{3 / 2}$ doublets at $55.5 \mathrm{eV}$ and $54.7 \mathrm{eV}$ for Se and

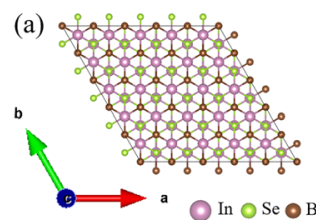

(b)

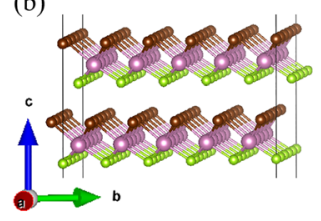

d)

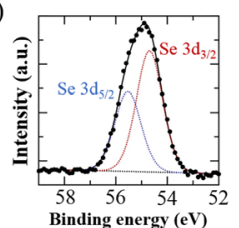

(c)

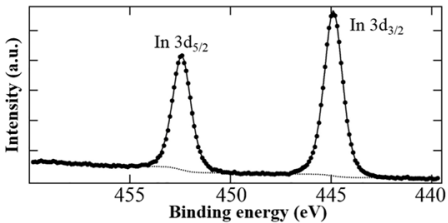

(e)

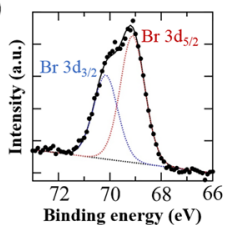

(f)

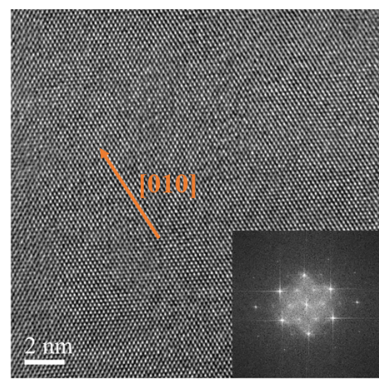

(g)

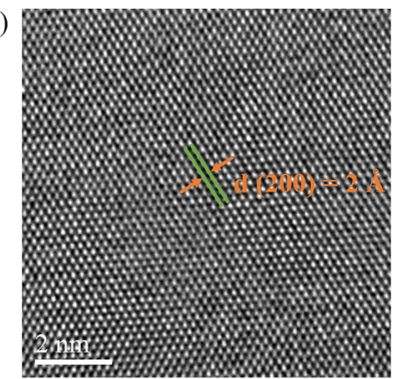

FIG. 1. InSeBr structures and characterization. (a) Top view and (b) side view of the crystal structure of few-layer $\mathrm{InSeBr}$. In atoms are purple, Se atoms are green, and $\mathrm{Br}$ atoms are brown. XPS spectra of (c) In, (d) Se, and (e) $\mathrm{Br}$ in InSeBr sheets. (f) HRTEM image of a few-layer $\mathrm{InSeBr}$ flake from the [001] zone axis, indicating the [010] preferential crystal orientation. Inset: corresponding fast Fourier transformation image. (g) The enlarged HRTEM image.
69.1 $\mathrm{eV}$ and $70.2 \mathrm{eV}$ for Br [Figs. 1(d) and 1(e)]. ${ }^{45,46}$ High-resolution transmission electron microscopy (HRTEM) imaging was performed to inspect the crystal quality and orientation of the layered $\mathrm{InSeBr}$ (see supplementary material S2). The HRTEM image and corresponding fast Fourier transformation (FFT) pattern in Fig. 1(f) show that the layered $\mathrm{InSeBr}$ has a good single-crystalline structure. The inter-planar distance $(2 \AA)$ of the $(200)$ plane can be recognized and measurable in Fig. $1(\mathrm{~g})$, which is in good agreement with the optimized in-plane lattice constants in the first-principles calculation.

For the InSeBr crystal, it belongs to the $D_{3 d}^{5}(R \overline{3} m)$ space group. ${ }^{43}$ According to group theory, the symmetries of Raman active modes and the corresponding Raman tensors (R) are described as follows: ${ }^{47}$

$$
A_{1 g}:\left(\begin{array}{ccc}
a & 0 & 0 \\
0 & a & 0 \\
0 & 0 & b
\end{array}\right) ; \quad E_{g}:\left(\begin{array}{ccc}
0 & c & d \\
c & 0 & 0 \\
d & 0 & 0
\end{array}\right)\left(\begin{array}{ccc}
c & 0 & 0 \\
0 & -c & d \\
0 & d & 0
\end{array}\right) .
$$

For the non-resonant Raman scattering intensity of a Raman active mode in a crystal, it can be calculated within the Placzek approximation and expressed by the Raman tensor as $I \propto\left|e_{i} \cdot R \cdot e_{s}\right|^{2}$, where $e_{i}$ and $e_{s}$ are the polarization vectors of the incident and scattered light, respectively. ${ }^{47}$ In the back-scattering configuration, $e_{i}$ and $e_{s}$ are within the $x y$ plane. Using the tabulated Raman tensors, we can know that the intensity of $\mathrm{A}_{1 \mathrm{~g}}$ mode is maximum (minimum) when the incident light is parallel (perpendicular) to the scattered light, while the intensity of $\mathrm{E}_{\mathrm{g}}$ is independent of the angle $\theta$ between $e_{i}$ and $e_{s}$.

2D InSeBr flakes were mechanically exfoliated onto a silicon wafer with a $285 \mathrm{~nm}$ silica layer (see supplementary material S3). ${ }^{1}$ An optical microscopy image of few-layer InSeBr is shown in Fig. S3 (supplementary material) with a strongly layer-dependent optical contrast, which indicates the characteristics of the layered structure. Figure 2(a) shows the topography of atomic force microscopy (AFM) scanning (see supplementary material S3). The height profiles indicate that the thickness of the majority part of the flake is $\sim 6 \mathrm{~nm}$, corresponding to $\sim 10$ layers [Fig. 2(b)]. In order to confirm the Raman fingerprints of InSeBr, we first performed the unpolarized Raman scattering with three laser excitation wavelengths $(488,532$, and $633 \mathrm{~nm})$ in ambient conditions, as shown in Fig. 2(c). Three non-resonant Raman modes are evidently observed (indicated by the blue dashed vertical lines). Through

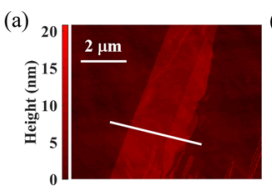

(b)

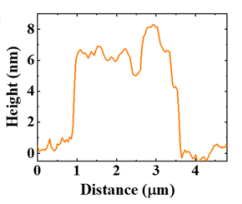

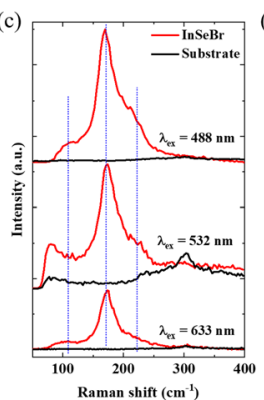

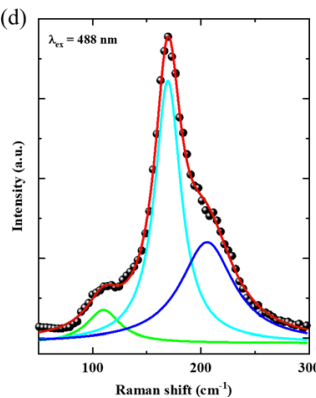

FIG. 2. InSeBr flake and its unpolarized Raman response at different wavelengths (a) AFM image of the InSeBr flake. (b) Height profiles taken along the white line in Fig. 2(a). (c) Unpolarized Raman spectra of $\mathrm{InSeBr}$ and the $\mathrm{SiO}_{2}$ substrate obtained with 488, 532, and $633 \mathrm{~nm}$ laser excitation, respectively. The spectra are normalized and vertically offset for clarify. (d) The Lorentz fitting of the unpolarized Raman spectra of $\mathrm{InSeBr}$ with $488 \mathrm{~nm}$ laser excitation. 
Lorentz fitting of the results at $488 \mathrm{~nm}$ [Fig. 2(d)] and $633 \mathrm{~nm}$ [Fig. S4] (supplementary material), it can be known that these three peaks are identified at 111,170 , and $210 \mathrm{~cm}^{-1}$, respectively.

To further clarify the symmetry of phonons, we performed the linear polarization resolved Raman scattering measurements. More details on the measurement methods can be found in supplementary material S4. Figure 3(a) presents the Raman spectra excited by linearly polarized light and collected in co-polarized (linearly parallel, red) or cross-polarized (linearly perpendicular, black) geometry. The evolution of Raman intensities with the angle $\theta$ between $e_{i}$ and $e_{s}$ is presented in Fig. 3(b). For the mode of $111 \mathrm{~cm}^{-1}$, the intensities are independent of the angle $\theta$, indicating the symmetry of $\mathrm{E}_{\mathrm{g}}$. In stark contrast, the intensities of the other two modes $\left(170\right.$ and $210 \mathrm{~cm}^{-1}$ ) are strongly dependent on the angle $\theta$ and exhibit cosine behaviors (red lines), demonstrating the $\mathrm{A}_{\mathrm{g}}$ symmetry.

After the identification of lattice dynamics in InSeBr, we performed temperature-dependent measurements (see supplementary material S4) to investigate the coupling between phonons and other elementary excitations, which provide a firm basis for many physical phenomena, such as the formation of Cooper pairs, carrier dynamics, and spin-Seebeck effect. ${ }^{29,37,38,48}$ Since $A_{1 g}$ of $170 \mathrm{~cm}^{-1}$ possesses the largest intensity, we focus on this mode to study the temperature effects. Figure 4(a) shows the temperature-dependent Raman spectra. The temperature-driven evolution of phonon frequencies $(\omega)$ and the full width at half maximum (FWHM) for the $\mathrm{A}_{1 \mathrm{~g}}$ mode at $\sim 170 \mathrm{~cm}^{-1}$ are presented in Figs. 4(b) and 4(c), respectively. Usually, the temperature-dependent phonon frequencies (linewidths) can be described in terms of the anharmonic model and increase (decrease) with decreasing temperature. ${ }^{29,49}$ For the phonon frequencies, both the $170 \mathrm{~cm}^{-1}$ mode of InSeBr and $520 \mathrm{~cm}^{-1}$ mode of silicon exhibit the monotonous blue-shift when temperature decreases, in fair agreement with the anharmonic model. For the FWHM, the $520 \mathrm{~cm}^{-1}$ mode of silicon can also be well described with the anharmonic model and decreases with decreasing temperature. In stark contrast, the FWHM for the $170 \mathrm{~cm}^{-1}$ mode of InSeBr increases with decreasing
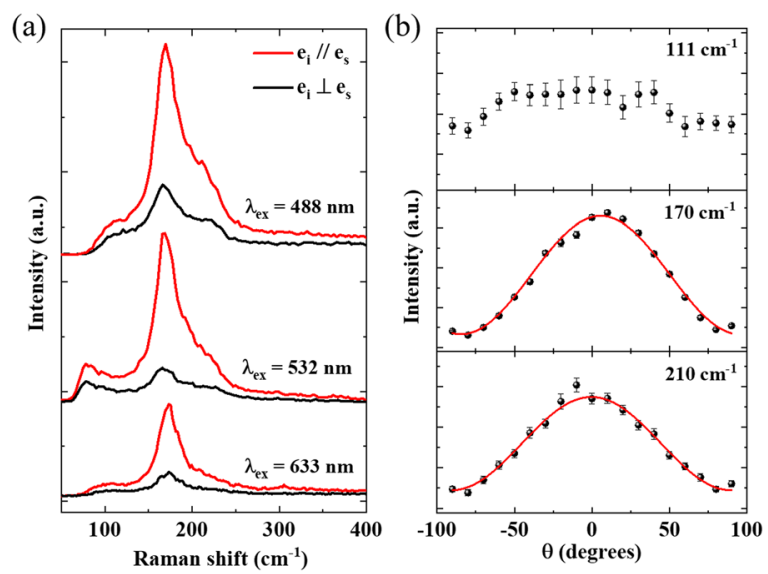

FIG. 3. Linearly polarized Raman responses of InSeBr. (a) Raman spectra of InSeBr obtained in the parallel and cross polarization conditions with 488, 532, and $633 \mathrm{~nm}$ excitation. (b) Peak intensity changes with the angle between incident (wavelength of $488 \mathrm{~nm}$ ) and scattered light polarization. The spectral resolution is about $1 \mathrm{~cm}^{-1}$. All the spectra are normalized and vertically offset for clarify.
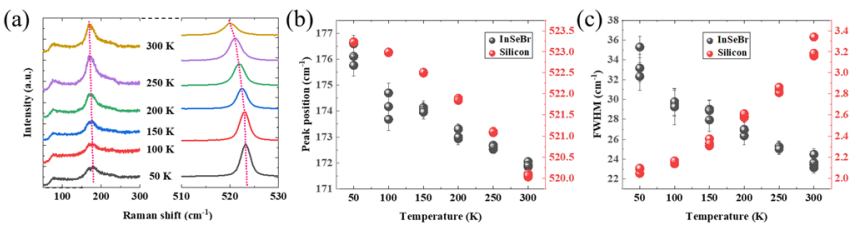

(d)

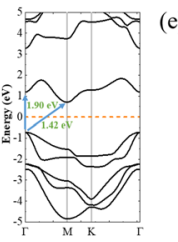

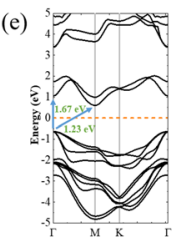

(g)

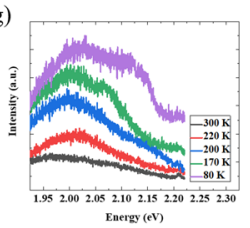

FIG. 4. (a) Temperature-dependent Raman spectra of the $\mathrm{InSeBr}$ flake and the silicon substrate reference. (b) Raman peak positions and (c) FWHM of the $170 \mathrm{~cm}^{-1}$ Raman mode of InSeBr (left vertical axis) and $520 \mathrm{~cm}^{-1}$ Raman mode of the silicon reference (right vertical axis) as a function of temperature. (d) Band structures of monolayer, (e) bilayer, and (f) bulk $\mathrm{InSeBr}$. (g) PL spectra of $\mathrm{InSeBr}$ at different temperatures.

temperature, strongly diverging from the standard anharmonic model. This phenomenon indicates another phonon relaxation pathway besides the symmetric anharmonic decay (i.e., decay into a pair of acoustic modes with identical frequencies and opposite momenta) and a strong coupling between the phonon and other elementary excitations. Since $\mathrm{InSeBr}$ is a non-magnetic material, such anomalous temperature-dependent FWHM behavior cannot come from the magnetic elementary excitations. . $^{29,48}$

Due to the reduced screening effect in 2D materials, the coupling between phonons and excitons would be significantly enhanced. ${ }^{38,40}$ We expect that the anomalous phenomenon of FWHM for the $170 \mathrm{~cm}^{-1}$ mode of InSeBr may stem from the exciton-phonon interaction, which can lead to the enhancement of FWHM. ${ }^{50}$ To confirm this point of view, we first performed first-principles calculations. A detailed description of the calculation methods is given in supplementary material S5. Figures 4(d)-4(f) show the electronic band structure of the monolayer, bilayer, and bulk InSeBr. For the monolayer, the direct bandgap at the $\Gamma$ point is $1.90 \mathrm{eV}$ and the indirect bandgap between $\Gamma$ and $\mathrm{M}$ points is $1.42 \mathrm{eV}$. Therefore, monolayer $\mathrm{InSeBr}$ is an indirect bandgap semiconductor, and the difference between direct and indirect bandgaps is $0.48 \mathrm{eV}$. With the increasing layer number, the direct bandgap, indirect bandgap, and bandgap differences for the bilayer (bulk) are $1.67 \mathrm{eV}(1.47 \mathrm{eV}), 1.23 \mathrm{eV}(1.34 \mathrm{eV})$, and $0.44 \mathrm{eV}$ $(0.13 \mathrm{eV})$, respectively. Evidently, the bandgap difference between direct and indirect bandgaps decreases with the increasing layer number, being akin to the case of InSe. ${ }^{24,51}$ As a consequence, multi-layer $\mathrm{InSeBr}$ is a nearly direct bandgap semiconductor and would harbor strong exciton-phonon coupling. Figure $4(\mathrm{~g})$ shows the temperaturedependent PL spectra. A prominent peak around $2 \mathrm{eV}$ can be clearly observed, which corresponds to the direct bandgap and is in good agreement with the first-principles theoretical prediction and the reflection spectrum in Fig. S6 (see supplementary material S6). Remarkably, the PL intensity increases with the decreasing temperature, leading to the significantly enhanced exciton-phonon interactions and FWHM of phonons.

In summary, we investigated the Raman fingerprints and exciton-phonon coupling of few-layer ternary $\mathrm{InSeBr}$ flakes by 
theoretical calculations and experimental studies. InSeBr is a layered semiconductor with a layer-dependent bandgap in the visible region ranging from $2.04 \mathrm{eV}$ (bulk) to $2.12 \mathrm{eV}$ (monolayer). Three Raman modes (two $A_{1 g}$ at $170 \mathrm{~cm}^{-1}$ and $210 \mathrm{~cm}^{-1}$ and one $E_{g}$ at $111 \mathrm{~cm}^{-1}$ ) are confirmed by polarization-dependent Raman spectra with different laser wavelengths $(488 \mathrm{~nm}, 532 \mathrm{~nm}$, and $633 \mathrm{~nm})$. Temperaturedependent Raman scattering shows that the FWHM of phonons in InSeBr strongly diverges from the standard anharmonic model. In conjunction with theoretical calculations and temperature-dependent PL spectra, we uncover the strong exciton-phonon coupling in fewlayer InSeBr flakes. This work exhibits a detailed study of the phonon properties and exciton-phonon interaction, paving the way toward the understanding and engineering of potential optoelectronic applications on $2 \mathrm{D}$ ternary semiconductors.

See the supplementary material for XPS characterization, HRTEM characterization, sample preparation, AFM characterization, Raman and PL characterization, theoretical calculations, and reflection characterization.

We thank Jiming Zheng (Northwest University, China) for computation service and the provision of facilities of Aalto University at the OtaNano-Nanomicroscopy Center (Aalto-NMC). This work was supported by the International Science and Technology Cooperation Project (No. 2014DFR10780), the National Natural Science Foundation of China (No. 11874299), the Key Industrial Chain Project of Shaanxi Province (No. 2018ZDCXL-GY-08-05), the Military-civilian Integration Dualmentorship Project of Shaanxi Province (No. 2018JM1059), Academy of Finland (Grant Nos. 295777, 312297, 319218, and 314810), Academy of Finland Flagship Programme (Grant No. 320167, PREIN), the European Union's Horizon 2020 research and innovation program (Grant No. 820423, S2QUIP), ERC (Grant No. 834742), and the financial support from the China Scholarship Council (No. 201706970027).

There are no conflicts to declare.

\section{REFERENCES}

${ }^{1}$ K. S. Novoselov, A. K. Geim, S. V. Morozov, D. Jiang, Y. Zhang, S. V. Dubonos, I. V. Grigorieva, and A. A. Firsov, Science 306, 666 (2004).

${ }^{2}$ K. S. Novoselov, A. Mishchenko, A. Carvalho, and A. H. C. Neto, Science 353, aac9439 (2016).

${ }^{3}$ K. F. Mak, D. Xiao, and J. Shan, Nat. Photonics 12, 451 (2018).

${ }^{4}$ X. Zhang, X. F. Qiao, W. Shi, J. B. Wu, D. S. Jiang, and P. H. Tan, Chem. Soc. Rev. 44, 2757 (2015).

${ }^{5}$ Z. Sun, A. Martinez, and F. Wang, Nat. Photonics 10, 227 (2016).

${ }^{6}$ C. Jin, E. Y. Ma, O. Karni, E. C. Regan, F. Wang, and T. F. Heinz, Nat. Nanotechnol. 13, 994 (2018)

${ }^{7}$ C. Gong and X. Zhang, Science 363, eaav4450 (2019).

${ }^{8}$ X. Xu, W. Yao, D. Xiao, and T. F. Heinz, Nat. Phys. 10, 343 (2014).

${ }^{9}$ P. Luo, F. Zhuge, Q. Zhang, Y. Chen, L. Lv, Y. Huang, H. Li, and T. Zhai, Nanoscale Horiz. 4, 26 (2019).

${ }^{10}$ K. F. Mak, K. He, J. Shan, and T. F. Heinz, Nat. Nanotechnol. 7, 494 (2012).

${ }^{11}$ H. Zeng, J. Dai, W. Yao, D. Xiao, and X. Cui, Nat. Nanotechnol. 7, 490 (2012).

${ }^{12}$ L. Du, Q. Zhang, B. Gong, M. Liao, J. Zhu, H. Yu, R. He, K. Liu, R. Yang, D. Shi, L. Gu, F. Yan, G. Zhang, and Q. Zhang, Phys. Rev. B 97, 115445 (2018).

${ }^{13}$ L. Du, Q. Zhang, T. Zhang, Z. Jia, J. Liang, G. B. Liu, R. Yang, D. Shi, J. Xiang, K. Liu, Z. Sun, Y. Yao, Q. Zhang, and G. Zhang, Phys. Rev. B 100, 161404 (2019).
${ }^{14}$ E. J. Sie, J. W. McIver, Y. H. Lee, L. Fu, J. Kong, and N. Gedik, Nat. Mater. 14, 290 (2015).

${ }^{15}$ J. Yan, Y. Zhang, P. Kim, and A. Pinczuk, Phys. Rev. Lett. 98, 166802 (2007).

${ }^{16}$ L. Li, Y. Yu, G. J. Ye, Q. Ge, X. Ou, H. Wu, D. Feng, X. H. Chen, and Y. Zhang, Nat. Nanotechnol. 9, 372 (2014).

${ }^{17}$ F. Xia, H. Wang, J. C. M. Hwang, A. H. C. Neto, and L. Yang, Nat. Rev. Phys. 1, 306 (2019).

${ }^{18}$ L. Tao, E. Cinquanta, D. Chiappe, C. Grazianetti, M. Fanciulli, M. Dubey, A. Molle, and D. Akinwande, Nat. Nanotechnol. 10, 227 (2015).

${ }^{19}$ F. Liu, C. C. Liu, K. Wu, F. Yang, and Y. Yao, Phys. Rev. Lett. 111, 066804 (2013).

${ }^{20}$ A. Splendiani, L. Sun, Y. Zhang, T. Li, J. Kim, C. Y. Chim, G. Galli, and F. Wang, Nano Lett. 10, 1271 (2010).

${ }^{21}$ G. B. Liu, D. Xiao, Y. Yao, X. Xu, and W. Yao, Chem. Soc. Rev. 44, 2643 (2015).

${ }^{22}$ X. Zhou, J. Cheng, Y. Zhou, T. Cao, H. Hong, Z. Liao, S. Wu, H. Peng, K. Liu, and D. Yu, J. Am. Chem. Soc. 137, 7994 (2015).

${ }^{23}$ X. Hu, P. Huang, K. Liu, B. Jin, X. Zhang, X. Zhang, X. Zhou, and T. Zhai, ACS Appl. Mater. Interfaces 11, 23353 (2019).

${ }^{24}$ D. A. Bandurin, A. V. Tyurnina, G. L. Yu, A. Mishchenko, V. Zólyomi, S. V. Morozov, R. K. Kumar, R. V. Gorbachev, Z. R. Kudrynskyi, S. Pezzini, Z. D. Kovalyuk, U. Zeitler, K. S. Novoselov, A. Patanè, L. Eaves, I. V. Grigorieva, V. I. Fal'ko, A. K. Geim, and Y. Cao, Nat. Nanotechnol. 12, 223 (2017).

${ }^{25}$ Z. Yang, W. Jie, C. H. Mak, S. Lin, H. Lin, X. Yang, F. Yan, S. P. Lau, and J. Hao, ACS Nano 11, 4225 (2017).

${ }^{26}$ T. Gao, Q. Zhang, L. Li, X. Zhou, L. Li, H. Li, and T. Zhai, Adv. Opt. Mater. 6, 1800058 (2018).

${ }^{27}$ J. Wu, H. Yuan, M. Meng, C. Chen, Y. Sun, Z. Chen, W. Dang, C. Tan, Y. Liu, J. Yin, Y. Zhou, S. Huang, H. Q. Xu, Y. Cui, H. Y. Hwang, Z. Liu, Y. Chen, B. Yan, and H. Peng, Nat. Nanotechnol. 12, 530 (2017).

${ }^{28}$ Y. Deng, Y. Yu, Y. Song, J. Zhang, N. Z. Wang, Z. Sun, Y. Yi, Y. Z. Wu, S. Wu, J. Zhu, J. Wang, X. H. Chen, and Y. Zhang, Nature 563, 94 (2018).

${ }^{29}$ L. Du, J. Tang, Y. Zhao, X. Li, R. Yang, X. Hu, X. Bai, X. Wang, K. Watanabe, T. Taniguchi, D. Shi, G. Yu, X. Bai, T. Hasan, G. Zhang, and Z. Sun, Adv. Funct. Mater. 29, 1904734 (2019).

${ }^{30}$ F. Wang, T. Gao, Q. Zhang, Z. Y. Hu, B. Jin, L. Li, X. Zhou, H. Li, G. V. Tendeloo, and T. Zhai, Adv. Mater. 31, 1806306 (2019).

${ }^{31}$ Z. Fei, B. Huang, P. Malinowski, W. Wang, T. Song, J. Sanchez, W. Yao, D. Xiao, X. Zhu, A. F. May, W. Wu, D. H. Cobden, J. H. Chu, and X. Xu, Nat. Mater. 17, 778 (2018).

${ }^{32}$ A. F. May, S. Calder, C. Cantoni, H. Cao, and M. A. McGuire, Phys. Rev. B 93, 014411 (2016).

${ }^{33}$ J. Li, Z. Wang, Y. Wen, J. Chu, L. Yin, R. Cheng, L. Lei, P. He, C. Jiang, L. Feng, and J. He, Adv. Funct. Mater. 28, 1706437 (2018).

${ }^{34}$ J. Wu, Y. Liu, Z. Tan, C. Tan, J. Yin, T. Li, T. Tu, and H. Peng, Adv. Mater. 29, 1704060 (2017).

${ }^{35}$ Q. Fu, C. Zhu, X. Zhao, X. Wang, A. Chaturvedi, C. Zhu, X. Wang, Q. Zeng, J. Zhou, F. Liu, B. K. Tay, H. Zhang, S. J. Pennycook, and Z. Liu, Adv. Mater. 31, 1804945 (2019).

${ }^{36}$ X. Jiang, Y. Han, H. Zhang, H. Liu, Q. Huang, T. Wang, Q. Sun, and Z. Li, ACS Appl. Mater. Interfaces 10, 43396 (2018).

${ }^{37}$ C. Jin, J. Kim, J. Suh, Z. Shi, B. Chen, X. Fan, M. Kam, K. Watanabe, T. Taniguchi, S. Tongay, A. Zettl, J. Wu, and F. Wang, Nat. Phys. 13, 127 (2017).

${ }^{38}$ L. Du, M. Liao, J. Tang, Q. Zhang, H. Yu, R. Yang, K. Watanabe, T. Taniguchi, D. Shi, Q. Zhang, and G. Zhang, Phys. Rev. B 97, 235145 (2018).

${ }^{39}$ L. Du, Y. Zhao, Z. Jia, M. Liao, Q. Wang, X. Guo, Z. Shi, R. Yang, K. Watanabe, T. Taniguchi, J. Xiang, D. Shi, Q. Dai, Z. Sun, and G. Zhang, Phys. Rev. B 99, 205410 (2019).

${ }^{40}$ C. M. Chow, H. Yu, A. M. Jones, J. Yan, D. G. Mandrus, T. Taniguchi, K. Watanabe, W. Yao, and X. Xu, Nano Lett. 17, 1194 (2017).

${ }^{41}$ X. Hu, J. Zheng, and Z. Ren, Front. Phys. 13, 137302 (2018).

${ }^{42}$ H. Hahn and W. Nickels, Z. Anorg. Allg. Chem. 314, 307 (1962).

${ }^{43}$ R. Kniep, A. Wilms, J. Beister, and K. Syassen, Z. Naturforsch. B 36, 1520 (1981).

${ }^{44}$ L. Wang and S. J. Hwu, Chem. Mater. 19, 6212 (2007).

${ }^{45}$ K. M. Beck, W. R. Wiley, E. Venkatasubramanian, and F. Ohuchi, Appl. Surf. Sci. 255, 9707 (2009). 
${ }^{46}$ Y. Huo, J. Zhang, M. Miao, and Y. Jin, Appl. Catal. B 111-112, 334 (2012).

${ }^{47}$ R. Loudon, Adv. Phys. 50, 813 (2001).

${ }^{48}$ J. Bardeen, L. N. Cooper, and J. R. Schrieffer, Phys. Rev. 108, 1175 (1957).

${ }^{49}$ M. Balkanski, R. F. Wallis, and E. Haro, Phys. Rev. B 28, 1928 (1983).
${ }^{50}$ A. Raja, M. Selig, G. Berghäuser, J. Yu, H. M. Hill, A. F. Rigosi, L. E. Brus, A. Knorr, T. F. Heinz, E. Malic, and A. Chernikov, Nano Lett. 18, 6135 (2018).

${ }^{51}$ M. B. Gisbert, D. A. Penares, J. Suh, F. Hidalgo, R. Abargues, P. J. R. Cantó, A. Segura, A. Cros, G. Tobias, E. Canadell, P. Ordejón, J. Wu, J. P. M. Pastor, and J. F. S. Royo, Nano Lett. 16, 3221 (2016). 